\title{
Modeling of $Q$ for Generalized Linear Solid
}

\author{
Liu Xuefeng ${ }^{a, *}$, Fan Youhua $^{b}$ And Chang Dongmei ${ }^{c}$ \\ ${ }^{a}$ School of Aeronautical Engineering, Civil Aviation University of China, Tianjin 300300, China \\ ${ }^{b}$ School of Science, Harbin Institute of Technology, Shenzhen 518055, China \\ ${ }^{c}$ Tianjin Key Laboratory of High Speed Cutting and Precision Machining, \\ Tianjin University of Technology and Education, Tianjin 300222, China
}

(Received May 9, 2018; revised version May 14, 2019; in final form January 16, 2020)

\begin{abstract}
Modeling a given quality factor $Q$ with a relaxation spectrum is important to time-domain simulations of wave propagation in a viscoelastic medium. In this paper, we present an optimal scheme of modeling the quality factor with a relaxation spectrum based on a generalized linear solid, which is available for both constant and frequency-dependent $Q$. A detailed study is conducted on the distribution of stress relaxation times and the error in the modeling scheme. From the study, we find that the error will be larger if the distribution range is too small or too wide, especially, the error is always larger if the distribution range equals the frequency range of interest. Since seeking the distribution range that yields the highest accuracy needs a series of calculation, for convenience, we suggest distributing the reciprocals of stress relaxation times logarithmically over the range from half the minimal frequency of interest to twice the maximal frequency of interest, which always yields a high accuracy. The value of the frequency-independent $Q$ has little effect on the error. Moreover, effect of the number of SLSs (short for standard linear solid, a spring and a dashpot in parallel in series with a spring) on the accuracy is studied. We find that employing at least 5 SLSs leads to a higher accuracy. With this scheme, we model the frequency-dependent $Q$ functions. The modeling result shows the validity of the scheme, and the error is larger when modeling some $Q$ functions that decrease with frequency.
\end{abstract}

DOI: 10.12693/APhysPolA.137.276

PACS/topics: viscoelastic, $Q$ factor, generalized linear solid, relaxation time

\section{Introduction}

Many media such as rock, soil, and metal are exposed on waves attenuation, so they should be considered viscoelastic. The quality factor $Q$ is often used to determine the efect of attenuation $[1,2]$. Larger quality factor $Q$ indicates smaller attenuation of the medium. The quality factor is of fundamental interest in many fields such as groundwater, oil exploration, and earthquake seismology.

Some laboratory experiments show that the quality factor $Q$ is independent of frequency over a broad bandwidth $[3,4]$. There are also some other reports suggesting that $Q$ can be frequency-dependent [5-7]. Determination of the quality factor seems to be relevant since it is an important parameter in many fields. However, it is not clear what is the correct form of $Q$, i.e., whether it should be assumed frequency independent [8], or frequency dependent [5].

Modeling a given quality factor $Q$ with a relaxation spectrum is important to time-domain modeling of viscoelastic medium [9-11]. It is also critical for the forward deduction [12] and inversion [13, 14] of surface waves in viscoelastic medium. Many efforts are made not only for the case of constant $Q$, but also for the case of frequencydependent $Q$. Liu et al. [15] studied the relaxation spectrum of linear inelastic solid. A relaxation spectrum

*corresponding author; e-mail: milan6@126.com was chosen to give a nearly constant $Q$. In 1984, Day and Minster described an efficient scheme for incorporating general anelastic attenuation laws into time-marching methods for computing theoretical seismograms. In their paper [16], the relaxation spectrum for the case of frequency-independent $Q$ was obtained with the Padé approximate method. In 1995, Blanch et al. [17] presented a method for modeling constant $Q$ called $\tau$-method. This method is based on an explicit formula and appears to be more accurate than the Padé approximate method, since it was able to reduce computation time and memory requirements. In 2006, the other method for modeling constant $Q$ was obtained by Helthom et al. They used the nonlinear optimization Nelder-Mead algorithm [18, 19], thus presented even more accurate approach than the $\tau$ method [20]. In turn, Sabinin [21] used a pair of average relaxation times to model a constant $Q$ instead of a set of more relaxation times. In 2016, Blanc et al. [22] modeled a constant $Q$ with a nonlinear optimization in which both strain and stress relaxation times are involved. Their method may yields more accuracy for same number of relaxation times, but the optimization approach is more difficult. In 2018, Xie et al. studied the error of constant $Q$ modeling and seismic wave simulation [23].

Modeling frequency-dependent $Q$ is a bit more complicated. In 2004, Asvadurov et al. [24] presented a numerical scheme to model a frequency-dependent $Q$ by modifying the scheme for the case of constant $Q$ proposed by Emmerich and Korn [25]. In their paper one can use an approximated formula to model a constant $Q$. Liu and Archuleta derived an empirical formula to interpolate the weight coefficients for modeling a frequency-dependent $Q$ 
and for modeling the frequency-dependent $Q$ with simulated annealing algorithm [26]. Both attempts, however, are based on a generalized Maxwell solid, while there are no studies on modeling frequency-dependent $Q$ based on a generalized linear solid. Moreover, relevant aspects, like how to choose the initial value when modeling of constant or frequency-dependent $Q$, have not been studying in detail at all.

In this paper, we present an optimal scheme of modeling both constant and frequency-dependent $Q$ for generalized linear solid. We also give a detailed study on the distribution of stress relaxation times and the error in the modeling scheme.

\section{Generalized linear solid model}

We refer to the system consisting of a spring and a dashpot in series, in parallel with a spring, and call it SLS. As shown in Fig. 1, the generalized linear solid model consists of an array of SLSs. It is widely used in the simulation of wave propagation in viscoelastic media $[9,10,27,28]$.

For the $l$-th $(l=1, . ., L)$ SLS the complex stress modulus can be written as

$$
M_{l}(\omega)=M_{R l}\left(\frac{1+\mathrm{i} \omega \tau_{\varepsilon l}}{1+\mathrm{i} \omega \tau_{\sigma l}}\right) .
$$

The corresponding relaxed stress modulus, the stress relaxation times and the strain relaxation times are

$$
M_{R l}=\frac{k_{1 l} k_{2 l}}{k_{1 l}+k_{2 l}}, \quad \tau_{\sigma l}=\frac{\eta_{l}}{k_{1 l}+k_{2 l}}, \quad \tau_{\varepsilon l}=\frac{\eta_{l}}{k_{2 l}} .
$$

Since

$$
\sigma_{l}=M_{l} \varepsilon,
$$

the stress and strain of the medium yield

$$
\sigma=\sum_{l=1}^{L} M_{l} \varepsilon=\sum_{l=1}^{L} M_{R l}\left(\frac{1+\mathrm{i} \omega \tau_{\varepsilon l}}{1+\mathrm{i} \omega \tau_{\sigma l}}\right) \varepsilon .
$$

Now, assuming that

$$
M_{R l}=\frac{M_{R}}{L}, \quad(l=1, \ldots, L),
$$

the complex stress modulus of the medium yields

$$
M(\omega)=\frac{M_{R}}{L} \sum_{l=1}^{L}\left(\frac{1+\mathrm{i} \omega \tau_{\varepsilon l}}{1+\mathrm{i} \omega \tau_{\sigma l}}\right) .
$$

The quality factor $Q[29]$ is usually defined as

$$
Q(\omega)=\frac{\operatorname{Re}(M(\omega))}{\operatorname{Im}(M(\omega))} .
$$

Therefore, its relation with frequency at given relaxation time spectrum becomes

$$
Q(\omega)=\left(\sum_{l=1}^{L} \frac{1+\omega^{2} \tau_{\varepsilon_{l}} \tau_{\sigma l}}{1+\omega^{2} \tau_{\sigma l}^{2}}\right) /\left(\sum_{l=1}^{L} \frac{\omega\left(\tau_{\varepsilon l}-\tau_{\sigma l}\right)}{1+\omega^{2} \tau_{\sigma l}^{2}}\right) .
$$

Using (8), we can obtain the relaxation time spectrum for a given $Q$ function of a medium $Q(\omega)$ with optimization methods. The objective function can be written as

$$
\Phi=\left|Q_{n}(\omega)-Q(\omega)\right|_{2},
$$

where the function $Q_{n}(\omega)$ is the estimated $Q$ function, and $\|_{2}$ is the $l_{2}$-norm.

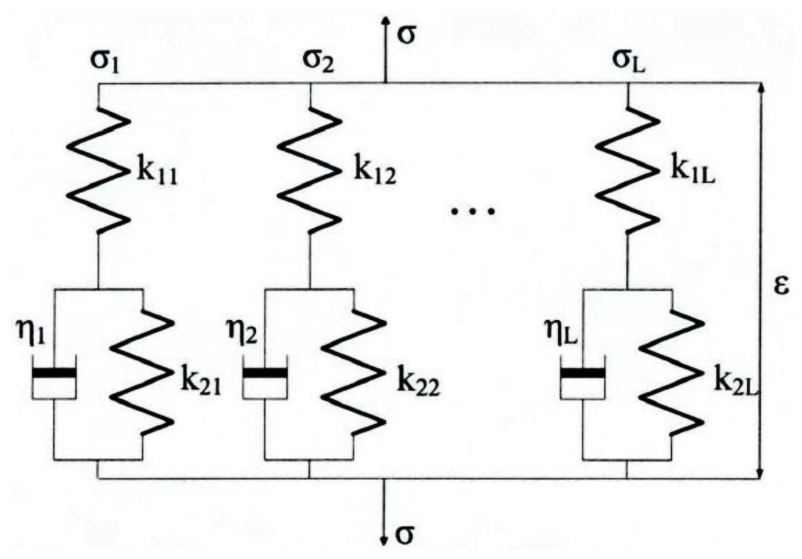

Fig. 1. The model of generalized linear solid.

A detailed study on modeling the relaxation time spectrum with optimization method will be given in this paper.

\section{Modeling of constant $Q$}

Minimizing (9) is a typical least squares problem. It can be solved with the Levenberg-Marquardt $(\mathrm{L}-\mathrm{M})$ method [30, 31]. Until now more studies are focused on modeling constant $Q$. It is a bit simpler as compare to modeling frequency-dependent $Q$, therefore, in this section we start from the problem of modeling constant $Q$.

We will proceed similarly to the schemes presented by other researchers $[17,20]$. At first we distribute the reciprocals of the stress relaxation times $\tau_{\sigma l}$ logarithmically, and then we seek for the strain relaxation times $\tau_{\varepsilon l}$ by minimizing (9). For example, to approximate model with $Q=20$, we employ 5 SLSs, i.e., $L=5$. The frequency range of interest varies from $2 \mathrm{~Hz}$ to $25 \mathrm{~Hz}$. The result obtained by Blanch et al. using $\tau$-method is shown in Fig. 2 and Table I (case 1) [17]. The maximal relative error and the average relative error is $3 \%$ and $0.92 \%$, respectively.

The stress relaxation times $\tau_{\sigma l}$ are chosen to be the same as those in the paper of Blanch et al. (case 1 in Table I) [17]. The initial value $\tau_{\varepsilon l}$ of the optimal problem is given by the $\tau$ method. The corresponding $Q$-curve, determined by minimizing (9) with $\mathrm{L}-\mathrm{M}$ method, is shown in Fig. 2. The relaxation times are given in Table I (case 2). The maximal relative error and the average relative error is $1.1 \%$ and $0.27 \%$, respectively. One can conclude, therefore, that the scheme using $\mathrm{L}-\mathrm{M}$ method allows to get higher accuracy than $\tau$-method.

Many researchers believe that distributing the reciprocals of the stress relaxation times $\tau_{\sigma l}$ logarithmically can lead to a good approximation [15, 17], how to choose the distribution range is still an open question. In former studies, the distribution range of frequency was as the frequency range of interest. In the paper of Blanch et al. [17], though stress relaxation times were presented, how to select them was not described clearly. 
TABLE 1

Approximation results assuming $L=5, f_{\min }=2[\mathrm{~Hz}]$ and $f_{\max }=25[\mathrm{~Hz}]$

\begin{tabular}{|c|c|c|c|c|c|c|c|}
\hline Case & $Q$ & $\begin{array}{l}\text { Max. rel. } \\
\text { error [\%] }\end{array}$ & $\begin{array}{l}\text { Avg. rel. } \\
\text { error [\%] }\end{array}$ & $a$ & $l$ & $\tau_{\sigma l}[\mathrm{~ms}]$ & $\tau_{\varepsilon l}[\mathrm{~ms}]$ \\
\hline \multirow{5}{*}{1} & \multirow{5}{*}{20} & \multirow{5}{*}{3.0} & \multirow{5}{*}{0.92} & & 1 & 265.26 & 281.2202 \\
\hline & & & & & 2 & 52.203 & 55.3440 \\
\hline & & & & & 3 & 10.273 & 10.8911 \\
\hline & & & & & 4 & 2.0218 & 2.1434 \\
\hline & & & & & 5 & 0.39789 & 0.4218 \\
\hline \multirow{5}{*}{2} & \multirow{5}{*}{20} & \multirow{5}{*}{1.1} & \multirow{5}{*}{0.27} & & 1 & 265.26 & 349.8503 \\
\hline & & & & & 2 & 52.203 & 66.9323 \\
\hline & & & & & 3 & 10.273 & 13.7355 \\
\hline & & & & & 4 & 2.0218 & 2.4971 \\
\hline & & & & & 5 & 0.39789 & 0.4101 \\
\hline \multirow{5}{*}{3} & \multirow{5}{*}{20} & \multirow{5}{*}{3.84} & \multirow{5}{*}{1.00} & \multirow{5}{*}{2} & 1 & 79.5775 & 104.7173 \\
\hline & & & & & 2 & 42.3217 & 46.6328 \\
\hline & & & & & 3 & 22.5079 & 23.7420 \\
\hline & & & & & 4 & 11.9704 & 11.9704 \\
\hline & & & & & 5 & 6.3662 & 9.1874 \\
\hline \multirow{5}{*}{4} & \multirow{5}{*}{20} & \multirow{5}{*}{0.18} & \multirow{5}{*}{0.05} & \multirow{5}{*}{2} & 1 & 159.1549 & 208.6572 \\
\hline & & & & & 2 & 59.8519 & 67.5090 \\
\hline & & & & & 3 & 22.5079 & 26.8829 \\
\hline & & & & & 4 & 8.4643 & 9.5733 \\
\hline & & & & & 5 & 3.1831 & 4.3648 \\
\hline \multirow{5}{*}{5} & \multirow{5}{*}{20} & & & & 1 & 159.1549 & 188.4429 \\
\hline & & & & & 2 & 59.8519 & 51.2968 \\
\hline & & 0.14 & 0.06 & 2 & 3 & 22.5079 & 28.5605 \\
\hline & & & & & 4 & 8.4643 & 9.9513 \\
\hline & & & & & 5 & 3.1831 & 3.7913 \\
\hline & & & & & 1 & 159.1549 & 168.9303 \\
\hline & & & & & 2 & 59.8519 & 61.2911 \\
\hline 6 & 100 & 0.16 & 0.05 & 2 & 3 & 22.5079 & 23.3110 \\
\hline & & & & & 4 & 8.4643 & 8.6693 \\
\hline & & & & & 5 & 3.1831 & 3.3854 \\
\hline & & & & & 1 & 79.5775 & 84.9235 \\
\hline & & & & & 2 & 42.3217 & 42.8154 \\
\hline 7 & 100 & 3.81 & 0.92 & 1 & 3 & 22.5079 & 22.8435 \\
\hline & & & & & 4 & 11.9704 & 11.9704 \\
\hline & & & & & 5 & 6.3662 & 6.8625 \\
\hline & & & & & 1 & 795.7747 & 795.7764 \\
\hline & & & & & 2 & 133.8328 & 144.0595 \\
\hline 8 & 100 & 0.71 & 0.39 & 10 & 3 & 22.5079 & 23.6800 \\
\hline & & & & & 4 & 3.7854 & 4.0828 \\
\hline & & & & & 5 & 0.6366 & 0.6366 \\
\hline & & & & & 1 & 159.1549 & 243.6722 \\
\hline & & & & & 2 & 59.8519 & 72.9740 \\
\hline 9 & Eq.(10) & 0.16 & 0.049 & 2 & 3 & 22.5079 & 29.6652 \\
\hline & & & & & 4 & 8.4643 & 10.2983 \\
\hline & & & & & 5 & 3.1831 & 4.9124 \\
\hline & & & & & 1 & 159.1549 & 164.4224 \\
\hline & & & & & 2 & 59.8519 & 68.3224 \\
\hline 10 & Eq.(11) & 11.31 & 2.35 & 2 & 3 & 22.5079 & 22.7804 \\
\hline & & & & & 4 & 8.4643 & 8.4643 \\
\hline & & & & & 5 & 3.1831 & 4.2698 \\
\hline
\end{tabular}

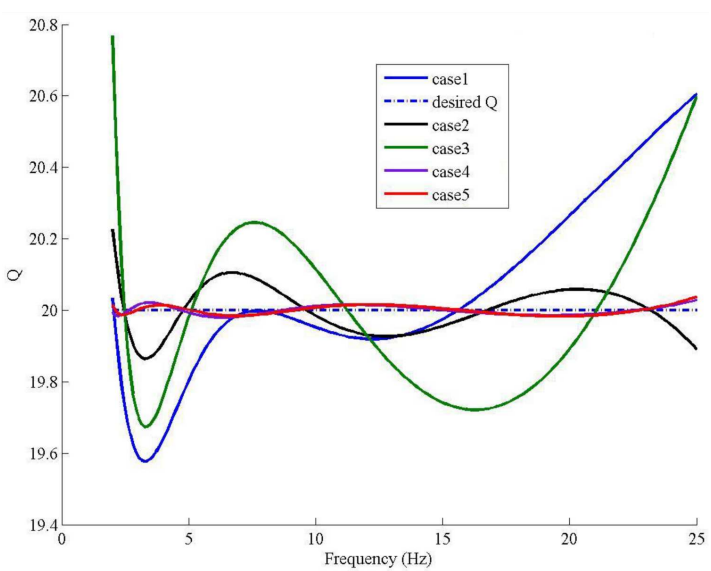

Fig. 2. Optimal approximation for $Q=20$.

That is why for the $Q=20$ model, we distribute the reciprocals of $\tau_{\sigma l}$ logarithmically over the frequency range of interest, and determine the initial value of $\tau_{\varepsilon l}$ with $\tau$-method. Next we minimize (9) with L-M method. The approximated $Q$ is shown in Fig. 2, and the values of relaxation times are given in Table I (case 3 ). The maximal relative error and the average relative error is $3.84 \%$ and $1.00 \%$, respectively. Note that the accuracy is lower than case 2 in which values of $\tau_{\sigma l}$ were the same as in [17]. Because there is a constraint in the optimization that $\tau_{\varepsilon l}$ should be larger than $\tau_{\sigma l}$, the accuracy is even lower than that of $\tau$-method in case 1 .

When comparing case 3 with case 2 , one can see that the distribution range of $\tau_{\sigma l}$ has a great influence on the accuracy. We enlarge the distribution range to $\left[\frac{1}{2} f_{\min }, 2 f_{\max }\right]$, where $f_{\min }$ and $f_{\max }$ are the minimal and maximal frequency, in the frequency range of interest, respectively. The approximated result is shown in Fig. 2, and the values of relaxation times are given in Table I (case 4). The maximal relative error and the average relative error is $0.18 \%$ and $0.05 \%$, respectively. Regarding the former cases, the accuracy in case 4 is significantly improved by distributing the reciprocals of $\tau_{\sigma l}$ logarithmically over $\left[\frac{1}{2} f_{\min }, 2 f_{\max }\right]$.

Helthom et al. presented also a method for modeling constant $Q$ [20]. In that paper, initial values of relaxation times are given by $\tau$ method, but at the same time both $\tau_{\sigma l}$ and $\tau_{\varepsilon l}$ are sought with one optimization algorithm, namely Matlab routine fminsearch. Here, we test the effect of the scheme. Again, let $Q=20$, and the reciprocals of $\tau_{\sigma l}$ is distributed logarithmically over $\left[\frac{1}{2} f_{\min }, 2 f_{\max }\right]$, and the initial $\tau_{\varepsilon l}$ is given by $\tau$ method. Then seek $\tau_{\sigma l}$ and $\tau_{\varepsilon l}$ simultaneously with Matlab routine fminsearch. The resulted $Q$ function is shown in Fig. 2, and the values of relaxation times are shown in Table I (case 5). The maximal relative error and the average relative error are $0.14 \%$ and $0.06 \%$, respectively. Similar to case 4 , the accuracy is still the same, with the result of seeking the strain relaxation times alone. Furthermore, although $\tau_{\sigma l}$ and $\tau_{\varepsilon l}$ are sought simultaneously, the result 


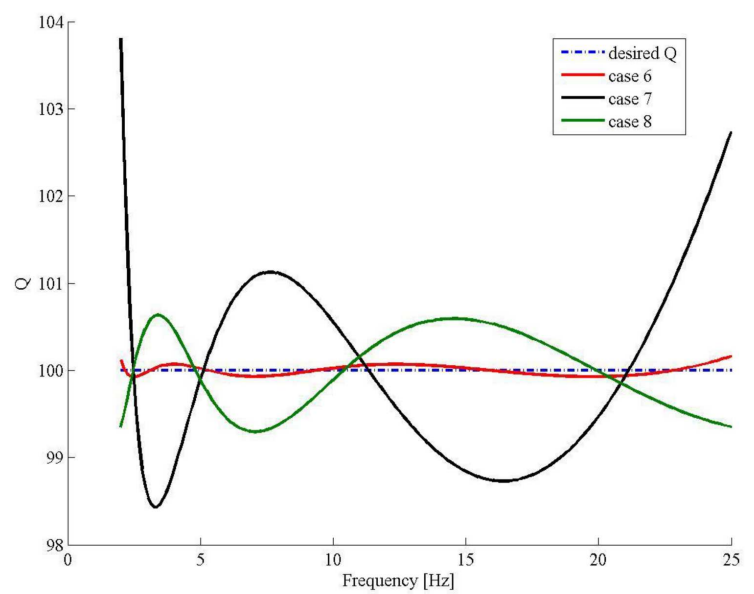

Fig. 3. Optimal approximation for $Q=100$.

always depends on the initial value. If the distribution range of $\tau_{\sigma l}$ is changed, like case 2 or 3 for example, the errors will be larger. This may be caused by the nonlinearity when seeking $\tau_{\sigma l}$ and $\tau_{\varepsilon l}$ simultaneously. Therefore, seeking $\tau_{\sigma l}$ and $\tau_{\varepsilon l}$ simultaneously is not better than the scheme of our paper.

The efficiency of our scheme can be tested for the larger values of $Q$. If the $Q=100$ over the frequency range of $2 \mathrm{~Hz}$ to $25 \mathrm{~Hz}$, the approximated result when the reciprocals of $\tau_{\sigma_{l}}$ are distributed logarithmically over the range of $\left[\frac{1}{2} f_{\min }, 2 f_{\max }\right]$ is shown in Fig. 3 , and the values of relaxation times are given in Table I (case 6). The maximal relative error is $0.16 \%$, and the average relative error is $0.05 \%$. Similar to case 4 , the accuracy is still very high. As shown in Fig. 3 and Table I (case 7), if the distribution range is $\left[f_{\min }, f_{\max }\right]$, the maximal relative error is $3.81 \%$, and the average relative error is $0.92 \%$. So also in the case of $Q=100$, the accuracy can be significantly improved by enlarging the distribution range to $\left[\frac{1}{2} f_{\min }, 2 f_{\max }\right]$.

Further enlarging the distribution range will not lead to higher accuracy. If we enlarge the distribution range to $\left[\frac{1}{10} f_{\min }, 10 f_{\max }\right]$, the result $Q$ function is shown in Fig. 3, and the values of relaxation times are shown in Table I (case 8). The maximal relative error is $0.71 \%$, and the average relative error is $0.39 \%$. Although the accuracy is still higher than the result with the distribution range of $\left[f_{\min }, f_{\max }\right]$, it is lower than the result when the distribution range is $\left[\frac{1}{2} f_{\min }, 2 f_{\max }\right]$.

Let us consider the general distribution range $\left[\frac{1}{a} f_{\min }, a f_{\max }\right]$. Figure 4 shows the variation of average relative errors with the value $a$ for setting $Q=20$, $Q=100$ and $Q=500$. We can observe that the average relative errors are lowest when $2<a<3$, while the absolute value of $Q$ has little effect on it. That is why in the latter part of this section we can just study the $Q=20$ model. When $a<1.5$, the average error increases rapidly. Figure 4 also shows the variation of average relative errors with the value $a$, when the frequency range of interest is from $2 \mathrm{~Hz}$ to $250 \mathrm{~Hz}$ and $Q=20$.

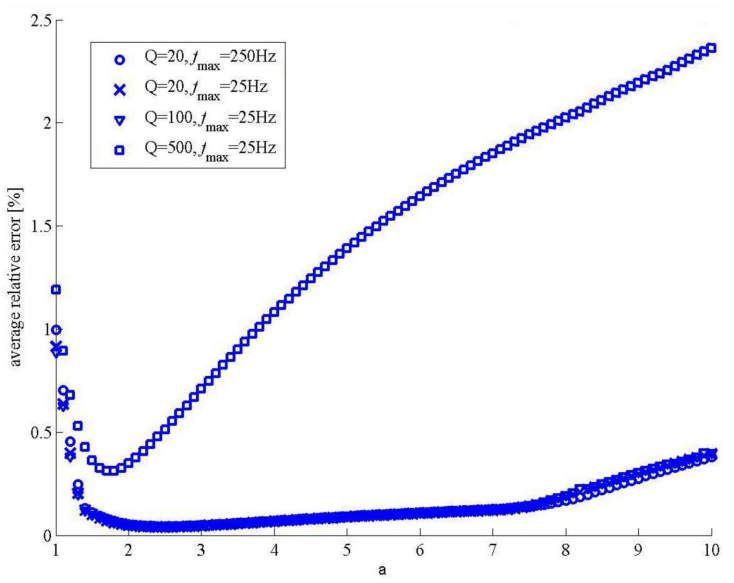

Fig. 4. The variation of average relative errors with the value $a, L=5$.

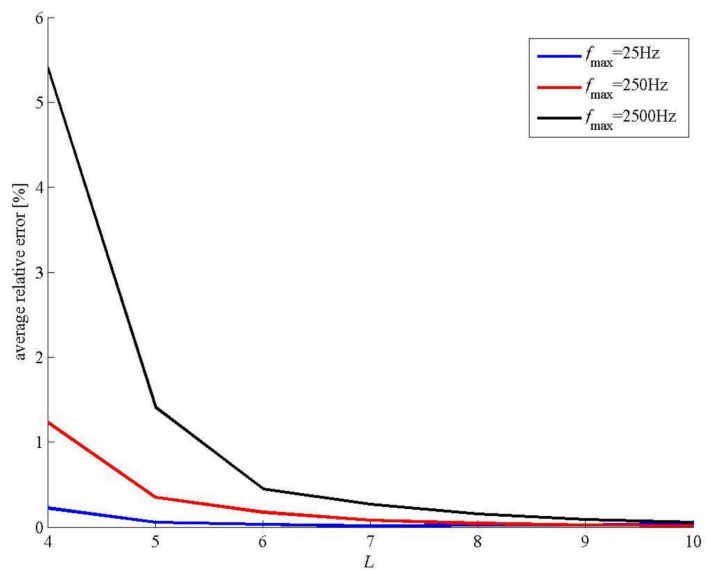

Fig. 5. The variation of average errors with the value $L$ when $Q=20$ and $a=2$.

When the frequency range of interest is larger, the average relative error is larger. In this case, the average relative errors are lowest when the value of $a$ is between 1.5 and 2 , when $a$ is smaller or larger, the average relative error increases rapidly. Furthermore, Fig. 4 shows that there is always a "best" value of $a$ that leads to a smallest average relative error, and the accuracies are high when $a$ is 2 for these cases.

In the above cases, $L$ is chosen to be 5 , but obviously the value of $L$ can affect the accuracy. Figures 5 shows the variation of average relative errors with the value of $L$ when $Q=20$ and $f_{\max }$ is $25 \mathrm{~Hz}, 250 \mathrm{~Hz}$, and $2500 \mathrm{~Hz}$, respectively. One can observe in Fig. 5 that there is a great difference between accuracies for $L=5$ and $L=4$. Therefore, setting $L>4$, i.e., employ at least 5 SLSs, seems to be reasonable suggestion. One may think that larger $L$ always leads to higher accuracy, however, it is opposite. When $L>7$, the accuracy gets a little bit lower. This is because minimization of (9) requires a constraint that $\tau_{\varepsilon l}$ should be larger than $\tau_{\sigma l}$. 

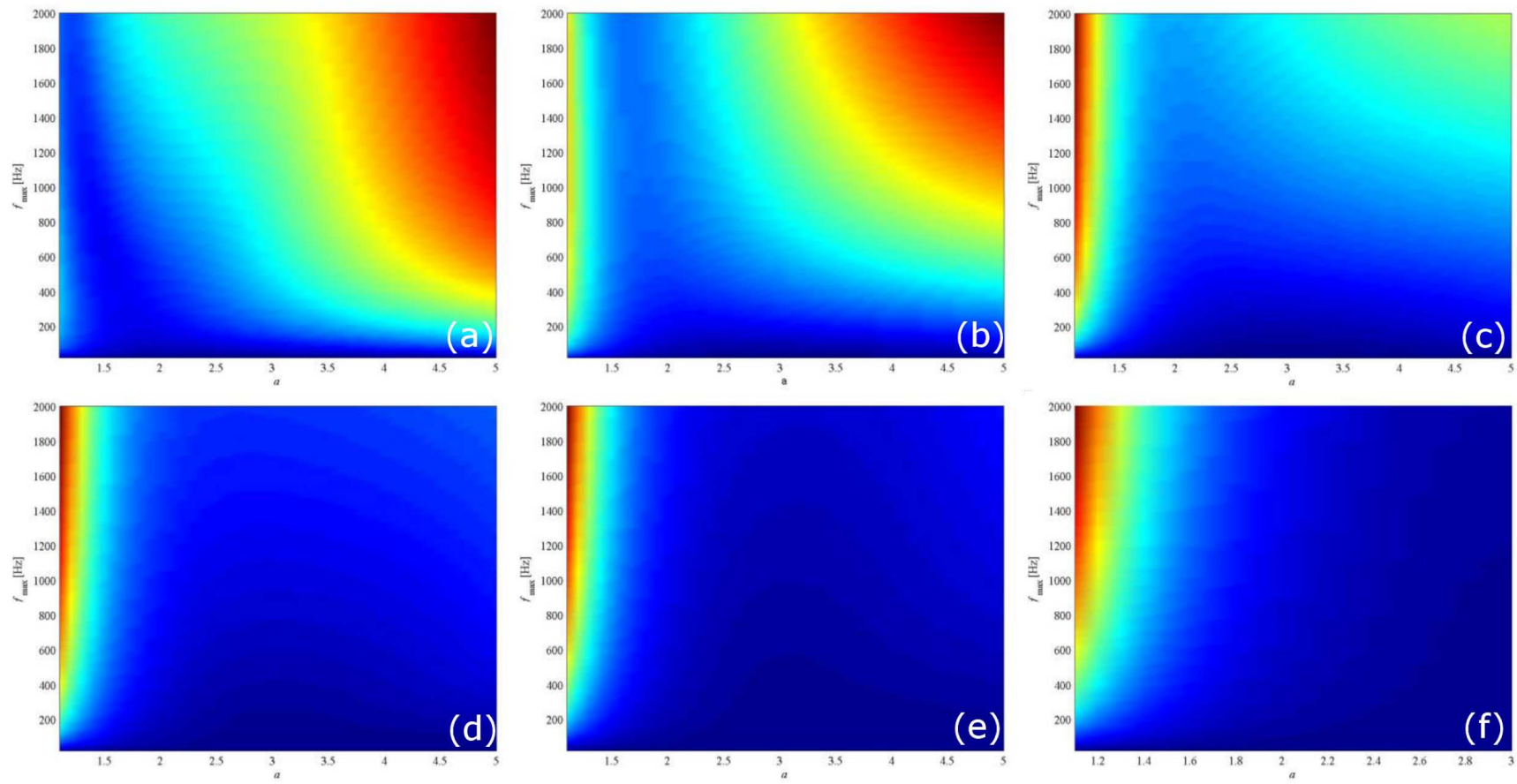

Fig. 6. Average relative error under different $a$ and $f_{\max }$, when (a) $L=5$, (b) $L=6$, (c) $L=7$, (d) $L=8$, (e) $L=9$, (f) $L=10$.

Figure 6 shows the average relative error for different values of $a$ and $f_{\max }$, when $L$ values varies in the range $5-10$, and $Q=20$. The colder color represents smaller average relative error, so indicating these values of $a$ leads to high accuracy under different conditions. The "best" value of $a$ is different for different sets of $f_{\max }$ and $L$ values. Therefore, series of calculations are required if one needs the highest accuracy. One can conclude that $a=2$ always leads to a high accuracy. All above figures confirm that a good and convenient choice is $a=2$.

\section{Modeling of frequency-dependent $Q$}

There are studies suggesting that $Q$ can be frequencydependent [5-7]. Several of them are based on a generalized Maxwell solid. In this section, we study the modeling of frequency-dependent $Q$ based on a generalized linear solid.

Two behaviour of frequency-dependent factor $Q$ can be distinquished typically: when $Q$ increases with frequency, and when $Q$ decreases with frequency. We assumed that these dependences are described by functions

$$
Q=10+2(0.2 \omega)^{0.2},
$$

and

$$
Q=50-2(0.02 \omega)^{2}
$$

in the range from $2 \mathrm{~Hz}$ to $25 \mathrm{~Hz}$. Rix and Meng obtained the damping ratio of polymethyl methacrylate (PMMA) and remolded kaolin experimentally, and the results are

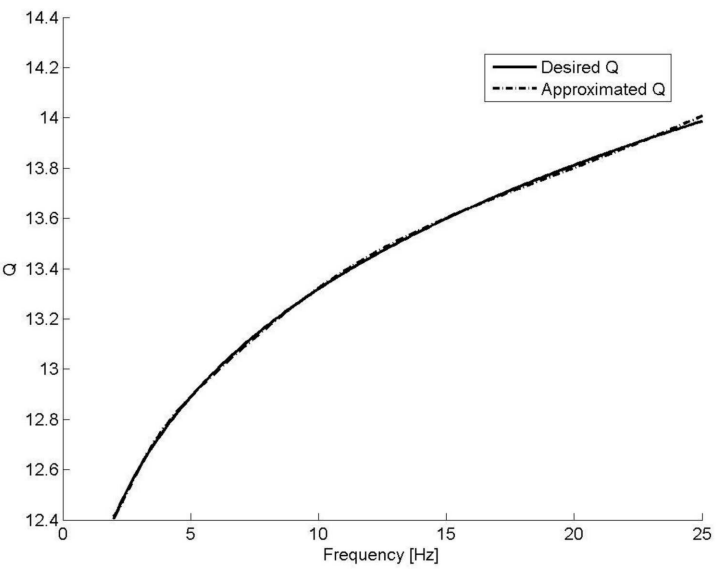

Fig. 7. Optimal approximation of the $Q$ function given by (10) for $L=5$. Average error is $0.049 \%$, while maximal error equals $0.16 \%$.

similar with these two functions [6]. For $L=5$ and $a=2$, the modeling result corresponding to (9) is shown in Fig. 7. The values of relaxation times are given in Table I (see case 9). The maximal relative error and the average relative error is is $0.16 \%$ and $0.049 \%$, respectively. Although the value of $Q$ varies with frequency, the modeling accuracy is still extremely high. This indicates that the scheme we used for frequency-dependent $Q$ is very efficient. Further, the accuracy depends on the value of $a$. In fact, the "best" value of $a$ is about 2.4 , however, $a=2$ is still a good choice, as shown in Fig. 8. 


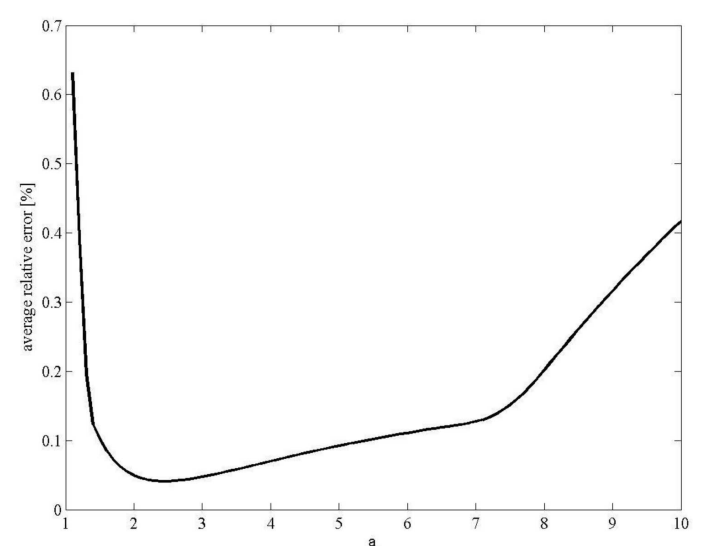

Fig. 8. The variation of average relative errors with the value $a$ when the $Q$ function is given by (10) for $L=5$.

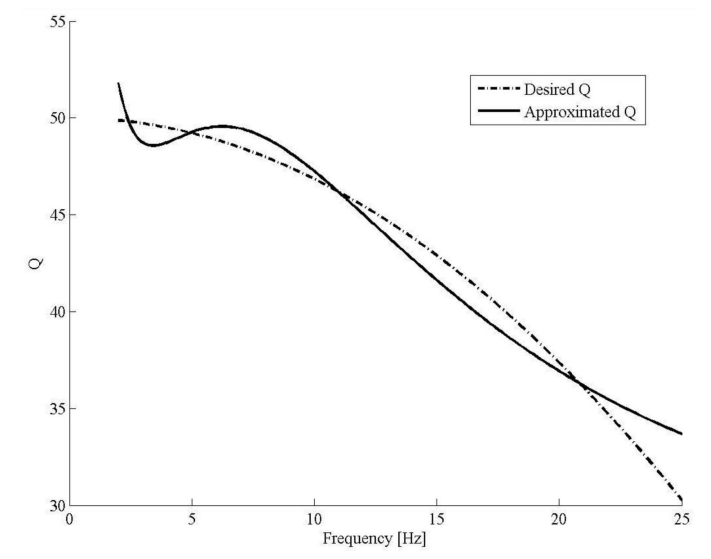

Fig. 9. Optimal approximation of the $Q$ function given by (11) for $L=5$. Average error is $2.3 \%$, while maximal error equals $11 \%$.

The result corresponds to (11) for $L=5$ is shown in Fig. 9. The maximal relative error and the average relative error are $11.31 \%$ and $2.35 \%$, respectively. The values of relaxation times are given in Table I (case 10). Note that the errors of case 9 are much larger. Our studies clearly show that the errors can be much larger when the $Q$-functions decrease with frequency similar to (11). Analogous observation were made by Liu and Archuleta [26], whose $Q$-function was modeled based on generalized Maxwell solid [26].

\section{Conclusions}

In this paper, we presented an optimal scheme of modeling the quality factor $Q$ with a relaxation spectrum based on generalized linear solid. Our approach becomes valid for both constant and frequency-dependent $Q$. We found that seeking both strain and stress relaxation times with one optimization algorithm at the same time are not more accurate than seeking strain relaxation times alone.
The accuracy appears to be always high when distributing the reciprocal of the stress relaxation times logarithmically over the range from half of the minimal frequency of interest to twice of the maximal frequency of interest. While the error will increase only if the distribution range is too narrow or too wide. Since seeking the distribution range that yields the highest accuracy needs a series of calculation, our suggestion is to distribute the reciprocals of stress relaxation times logarithmically from half of the minimal frequency of interest to twice of the maximal frequency of interest. The error appears to be much larger when the distribution range equals the frequency range of interest. The absolute value of the frequency-independent $Q$ has subtle effect on the error. Larger error is expected when the $Q$ function decreases with frequency. The study in this paper can be meaningful for time-domain simulation based on generalized linear solid.

\section{Acknowledgments}

This work was supported by the National Natural Sciences Foundation of China (Grant No. 41204042, 11602173) and Fundamental Research Funds for the Central Universities (Grant No. 3122018D017).

\section{References}

[1] L. Knopoff, G. MacDonald, Rev. Mod. Phys. 30, 1178 (1958).

[2] R.J. O'Connell, B. Budiansky, Geophys. Res. Lett. 5, 5 (1978).

[3] B.J. Mitchell, J. Geophys. Res. 80, 4904 (1975).

[4] D.H. Johnston, M.N. Toksoz, A. Timur, Geophysics 44, 691 (1979).

[5] Y.J. Jeng, T.S.Chen, Geophysics 64, 1608 (1999).

[6] G.J. Rix, J.W. Meng, Geotech. Test J. 28, 1 (2005).

[7] K.C. Meza-Fajardo, C.G. Lai, Geophys. J. Int. 171, 1247 (2007).

[8] J.H. Xia, R.D. Miller, C.B. Park, G. Tian, J. Appl. Geophys. 51, 121 (2002).

[9] N. Fan, L.F. Zhao, X.B. Xie, Z.X. Ge, Z.X. Yao, Geophys. J. Int. 206, 1539 (2016).

[10] M. Pasternak, Acta Phys. Pol. A 116, 371 (2009).

[11] E. Wang, J. Ba, Y. Liu, J. Geophys. , 1 (2018).

[12] Liu Xuefeng, Fan Youhua, Chang Dongmei, J. Harbin Inst. Technol. 49, 122 (2017).

[13] J. Xia, X. Yin, Y. Xu, J. Appl. Geophys. 95, 47 (2003).

[14] L. Gao, Y. Pan, G. Tian, J. Xia, Pure Appl. Geophys. 175, 2609 (2018).

[15] H.P. Liu, D.L. Anderson, H. Kanamori, Geophys. J. R. Astron. Soc. 47, 41 (1976).

[16] S.M. Day, J.B. Minster, Geophys. J. R. Astron. Soc. 78, 105 (1984).

[17] J.O. Blanch, O.A.J. Robertsson, W.S. William, Geophysics 60, 176 (1995). 
[18] J.A. Nelder, R. Mead, Computat. J. 7, 308 (1965).

[19] J.C. Lagarias, J.A. Reeds, M.H. Wright, P.E. Wright, Siam J. Optimiz. 9, 112 (1998).

[20] S. Hestholm, S. Ketcham, R. Greenfield, M. Moran, G. McMechan, Geophysics 71, T147 (2006).

[21] V. Sabinin, Geofis. Int. 51, 377 (2012).

[22] E. Blanc, D. Komatitsch, E. Chaljub, et al., J. Cryst. Growth 406, 18 (2016).

[23] Xie ZhiNan, Zheng YongLu, Zhang XuBin, Chin. J. Geophys. 61, 4007 (2018).

[24] S. Asvadurov, L. Knizhnerman, J. Pabon, Geophysics 69, 817 (2004).
[25] H. Emmerich, M. Korn, Geophysics 52, 1252 (1987).

[26] P.C. Liu, R.J. Archuleta, B. Seismol. Soc. Am. 96, 1352 (2006).

[27] J.M. Carcione, Geophysics 57, 781 (1992).

[28] K. Zhang, Y.H. Luo, J.H. Xia, C. Chen, Soil. Dyn. Earthq. Eng. 31, 1332 (2011).

[29] T. Bourbie, O. Coussy, B. Zinzner, Acoustics of Porous Media, Gulf Publ. Co., Houston 1987.

[30] K. Levenberg, Q. Appl. Math. 2, 164 (1944).

[31] D. Marquardt, Siam J. Appl. Math. 11, 431 (1963). 